\title{
Effets des rayonnements ionisants sur le facteur de bruit des transistors bipolaires aux moyennes fréquences
}

\author{
G. Blasquez et M. Roux-Nogatchewsky \\ Laboratoire d'Automatique et d'Analyse des Systèmes du Centre National de la Recherche Scientifique, \\ 7, avenue du Colonel-Roche, 31400 Toulouse, France
}

(Reçu le 28 mars 1979, accepté le 22 mai 1979)

\begin{abstract}
Résumé. - Un modèle liant les accroissements du facteur de bruit induits par le rayonnement à ceux du courant continu de base est proposé. Les propriétés principales du modèle sont comparées aux résultats des mesures effectuées sur des transistors bipolaires commandés par effet de champ. Il est établi théoriquement et expérimentalement que le rayonnement ionisant n'influence pas le facteur de bruit quand la résistance de source est inférieure à la résistance donnant le facteur de bruit minimum. Pour des résistances de source élevées, les centres de génération recombinaison induits à la surface par le rayonnement provoquent des accroissements du facteur de bruit proportionnels aux accroissements du courant de base. A bas niveau de polarisation, les accroissements correspondants du facteur de bruit minimum sont faibles. Ils résultent des effets de deux termes, l'un proportionnel au courant de base, l'autre proportionnel à sa racine carrée. De plus, la résistance de source donnant le facteur de bruit minimum décroît proportionnellement à l'inverse de la racine carrée du courant de base. L'étude de la sensibilité du bruit aux paramètres internes et externes du transistor indique que les accroissements du facteur de bruit sont amplifiés dans deux cas :
\end{abstract}

a) pour les faibles valeurs du courant émetteur quand la surface de base est appauvrie de porteurs majoritaires, et b) à fort niveau de courant quand l'effet de la défocalisation augmente l'injection des porteurs de charge à la périphérie de l'émetteur.

\begin{abstract}
A model connecting the radiation-induced noise figure shifts to the d.c. base current increases is proposed. The most significant features of the model are compared to measurements carried out on gate controlled bipolar transistors. It is established both theoretically and experimentally that ionizing radiation does not influence the noise figure behaviour when the source resistances are lower than the minimum noise figure resistance. For high source resistances, surface generation recombination centers induced by the ionizing radiation provoke noise figure increases in direct ratio to the increases of the base current. The corresponding shifts in the minimum noise figure have a small magnitude. They result from the effects of two terms, one proportional to the base current increases and the other proportional to its square root. Furthermore, the resistance source giving the minimum noise figure, decreases in inverse proportion to the base current increases.

The study of noise sensitivity to internal and external transistor parameters, indicates that radiation induced noise figure increases are magnified in two cases :
\end{abstract}

a) at low emitter current levels when the base surface is depleted and

b) at high emitter current levels, when crowding increases carrier injection at the emitter periphery.

1. Introduction. - D'une manière générale, les rayonnements ionisants (rayons $\mathrm{X}$, électrons de faible énergie) augmentent le courant inverse des jonctions p-n et modifient leur tension d'avalanche [1]. Dans les transistors bipolaires, l'effet prépondérant en régime statique est une diminution du gain en courant [1]. La plupart des auteurs [2-5] attribuent ces évolutions des caractéristiques électriques externes à la création d'une charge électrique positive dans la couche d'oxyde superficielle et à l'augmentation de la densité de centres de génération recombinaison situés à l'interface semiconducteur-oxyde.

A notre connaissance, peu de travaux ont porté sur le bruit de fond, mais à partir de considérations simples, on peut affirmer que celui des jonctions et transistors bipolaires est modifié par les rayonnements ionisants. En effet, d'après van Vliet [6], les centres de génération recombinaison créés par ce 
rayonnement dans la région superficielle de la jonction émetteur base doivent se comporter comme des sources supplémentaires de bruit. En outre, d'après les expériences de Martin, Blasquez et Caminade [7], effectuées sur des transistors bipolaires commandables par effet de champ, les variations du potentiel de surface, résultant de la création d'une charge positive, peuvent également provoquer des évolutions importantes du bruit de fond.

Dans ce qui suit, une étude plus approfondie des modifications apportées par le rayonnement ionisant aux propriétés de bruit des transistors bipolaires aux moyennes fréquences est effectuée. Les buts de cette étude sont :

a) d'établir un modèle du comportement du facteur de bruit et de ses principaux paramètres liant les évolutions du bruit à celles des caractéristiques statiques du transistor,

b) de rapporter des données expérimentales obtenues sur des transistors au silicium élaborés suivant la technologie planar,

c) de démontrer la validité du modèle et d'évaluer sa précision,

d) d'identifier la nature des sources de bruit effectivement créé par l'irradiation,

$e$ ) de déterminer les paramètres externes et internes des transistors qui influencent l'amplitude des évolutions du bruit observé après irradiation,

$f$ ) de proposer des solutions pour réduire la sensibilité au rayonnement des transistors dans les circuits électriques.

2. Analyse des effets des mécanismes physiques responsables des dégradations des caractéristiques statiques du transistor sur le bruit de fond. - 2.1 MODÈLE DE BRUIT DU TRANSISTOR AUX MOYENNES FRÉQUENCES. - En polarisation directe, les porteurs de charge injectés à la périphérie de l'émetteur se recombinent à la surface et sous le contact de base. En régime statique, ce mécanisme donne naissance à une composante du courant de base, notée dans ce qui suit $I_{\mathrm{L}}$, qui est reliée à la tension émetteur base $V_{\mathrm{BE}}$ par l'expression empirique [8] :

$$
I_{\mathrm{L}}=I_{\mathrm{L} 0} \exp \left(\frac{q V_{\mathrm{BE}}}{n_{\mathrm{L}} k T}\right)
$$

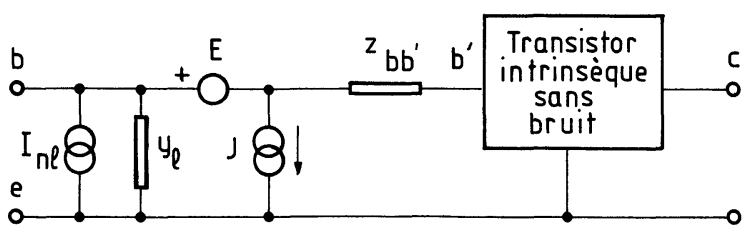

Fig. 1. - Circuit électrique équivalent au transistor bruyant prenant en compte les effets de surface.

[Transistor noise equivalent circuit taking into account surface effects.] où $q, k, T$ ont leur signification usuelle, $I_{\mathrm{L} 0}$ et $n_{\mathrm{L}}$ sont des coefficients caractéristiques de chaque transistor. Typiquement, $1<n_{\mathrm{L}} \leqslant 2$. En régime dynamique petit signal et en bruit de fond, les effets résultant de la recombinaison de ces porteurs peuvent être simulés par une admittance $y_{1}$ et un générateur de bruit $I_{n 1}$ placés en parallèle à l'entrée du transistor. Il a été indiqué dans [9] que $y_{1}$ et $I_{n 1}$ sont donnés aux basses et moyennes fréquences (typiquement jusqu'à quelques $\mathrm{MHz}$ ) par les relations suivantes :

$$
\begin{gathered}
y_{1}=\frac{q I_{\mathrm{L}}}{n_{\mathrm{L}} k T} \\
\left\langle I_{n 1} I_{n 1}^{*}\right\rangle=\frac{2 q I_{\mathrm{L}} \Delta f}{m_{\mathrm{L}}}
\end{gathered}
$$

où $m_{\mathrm{L}}$ est une constante empirique qui dépend du transistor considéré et qui est usuellement comprise entre les valeurs 1 et 1,33 environ.

Quand la présence de ces éléments est prise en compte, le circuit électrique équivalent au transistor bruyant peut se ramener à la configuration de la figure 1 où $z_{\mathrm{bb}^{\prime}}$ représente l'impédance de base, $E$ et $J$ sont les deux générateurs de bruit équivalents à l'ensemble des sources microscopiques de la zone active du transistor située sous l'émetteur.

A partir des résultats de la modélisation par circuit distribué de la zone active [10], on montre sans difficulté que :

$$
\begin{gathered}
\left\langle E E^{*}\right\rangle \simeq \Delta f\left[2 q I_{\mathrm{BA}} C_{n}\left|z_{\mathrm{bb}^{\prime}}\right|^{2}+4 k T\left(R_{n \mathrm{~b}}+\frac{r_{\mathrm{e}}}{2 \alpha_{0}}\right)\right] \\
\left\langle J J^{*}\right\rangle \simeq 2 q I_{\mathrm{BA}} \Delta f \\
\left\langle E J^{*}\right\rangle \simeq 2 q I_{\mathrm{BA}} \Delta f\left(z_{\mathrm{bb}^{\prime}}+\frac{r_{\mathrm{e}}}{\alpha_{0}}\right)
\end{gathered}
$$

où $I_{\mathrm{BA}}$ désigne la composante du courant de base associée à la zone active du transistor, $R_{n \mathrm{~b}}$ est la résistance de bruit associée à la base active,

$$
r_{\mathrm{e}}=k T /\left(q I_{\mathrm{E}}\right),
$$

$\alpha_{0}$ est le gain en courant continu dans le montage base commune du transistor intrinsèque, $C_{n} \simeq 4 / 3$ en première approximation. Les générateurs $E$ et $I_{n 1}$ d'une part, $J$ et $I_{n 1}$ d'autre part, ayant des origines physiques différentes ne sont pas corrélés :

$$
\left\langle E I_{n 1}^{*}\right\rangle=\left\langle J I_{n 1}^{*}\right\rangle=0 .
$$

Quand on place à l'entrée du transistor une source dont l'admittance interne est $y_{\mathrm{s}}$ et dont le générateur de bruit propre est $I_{n s}$, le facteur de bruit de l'ensemble source transistor est donné par la relation :

$$
F=1+\frac{\left\langle\left|I_{n 1}+J+E\left(y_{\mathrm{s}}+y_{1}\right)\right|^{2}\right\rangle}{\left\langle I_{n \mathrm{~s}} I_{n \mathrm{~s}}^{*}\right\rangle} .
$$


Dans ce qui suit, on admettra implicitement que la source est une résistance pure $R_{\mathrm{s}}$ et que son bruit est un bruit thermique donné par la formule bien connue de Nyquist-Johnson.

2.2 EFFETS DU RAYONNEMENT IONISANT SUR LE COURANT DE BASE [1] ET [11]. - La charge positive créée dans la couche d'oxyde et l'accroissement de la densité de centres de génération recombinaison provoqués par le rayonnement ont pour effet prépondérant, sur le comportement en régime direct de fonctionnement du transistor, une décroissance du gain en courant $h_{\mathrm{FE}}$. Pour l'essentiel, cette décroissance résulte d'une augmentation du courant de base $I_{\mathrm{B}}$ et plus précisément de sa composante $I_{\mathrm{L}}$. En accord avec Garric [11], l'accroissement de $I_{L}$ résulte à son tour de ceux de $I_{\mathrm{L} 0}$ et $n_{\mathrm{L}}$. Pour des doses absorbées de valeurs élevées, usuellement de l'ordre de $10^{7} \mathrm{~Gy}, n_{\mathrm{L}} \simeq 2$ et les accroissements de $I_{\mathrm{L} 0}$ tendent vers une limite qui est fonction des paramètres géométriques et technologiques du transistor. A notre connaissance, les relations existant entre ces paramètres et $I_{\mathrm{L} 0}$ n'ont pas encore été établies. En pratique avant irradiation $n_{\mathrm{L}} \geqslant 1,5$. Compte tenu de ce qui précède, les variations de $n_{\mathrm{L}}$ après irradiation sont relativement faibles. En première approximation nous admettrons dans ce qui suit que $n_{\mathrm{L}}$ reste constant quelle que soit la dose absorbée.

2.3 ANALYSE DES CONSÉQUENCES DES AUGMENTATIONS DU COURANT DE BASE SUR LE COMPORTEMENT EN BRUIT DE FOND. - D'après (2) et (3) les accroissements de $I_{\mathrm{L}}$ dus à l'irradiation provoquent des accroissements proportionnels de $y_{1}$ et de $\left\langle I_{n 1} I_{n 1}^{*}\right\rangle$. Par contre notons que $\left\langle E E^{*}\right\rangle,\left\langle J J^{*}\right\rangle$ et $\left\langle E J^{*}\right\rangle$ sont indépendants de $I_{\mathrm{L}}$. Pour des impédances de source faibles, le facteur de bruit est approximativement égal à :

$$
F \simeq 1+\frac{\left\langle E E^{*}\right\rangle}{4 k T R_{\mathrm{s}}}
$$

Les variations de $I_{\mathrm{L}}$ n'ont aucun effet significatif sur $F$.

Pour des impédances de source élevées ( $y_{\mathrm{s}}$ faible), (8) indique que :

$$
\begin{aligned}
& F \simeq 1+\frac{R_{\mathrm{s}}}{4 k T} \times \\
& \times\left[\left\langle J J^{*}\right\rangle+\left\langle I_{n 1} I_{n 1}^{*}\right\rangle+y_{1}^{2}\left\langle E E^{*}\right\rangle+2 y_{1} \operatorname{Re}\left\langle E J^{*}\right\rangle\right] .
\end{aligned}
$$

Pour la plupart des transistors polarisés à des courants inférieurs à quelques $\mathrm{mA}$, les deux derniers termes de (10) sont négligeables. Comme $\left\langle I_{n 1} I_{n 1}^{*}\right\rangle \propto I_{\mathrm{L}}$, on a $\Delta F \propto \Delta I_{\mathrm{L}}$. Pour des courants supérieurs à quelques $\mathrm{mA}$, les deux derniers termes de (10) ne sont pas nécessairement négligeables. Comme $y_{1}^{2} \propto I_{\mathrm{L}}^{2}$, $\Delta F \propto I_{\mathrm{L}}^{\gamma}$ avec $1<\gamma<2$. Cette augmentation de la sensibilité de $F$ à $I_{\mathrm{L}}$ est une conséquence de l'effet de défocalisation du courant émetteur [12]. Cet effet augmente l'injection des porteurs de charge à la périphérie de l'émetteur. L'influence des phénomènes de surface sur le comportement du transistor est alors renforcée.

Considérons maintenant le facteur de bruit au voisinage de son minimum. Dans le domaine des fréquences couvert par cet article, l'impédance de base se comporte comme une résistance et la partie imaginaire de $\left\langle E J^{*}\right\rangle$ est négligeable. Dans ce cas le facteur de bruit $F_{\min }$ prend la forme simplifiée suivante :

$$
\begin{aligned}
& F_{\min } \simeq \\
& \simeq 1+\frac{1}{2 k T \Delta f}\left\{\left\langle E J^{*}\right\rangle+y_{1}\left\langle E E^{*}\right\rangle+\left[\left\langle E E^{*}\right\rangle\left(\left\langle J J^{*}\right\rangle+\left\langle I_{n 1} I_{n 1}^{*}\right\rangle+y_{1}^{2}\left\langle E E^{*}\right\rangle+2 y_{1}\left\langle E J^{*}\right\rangle\right)\right]^{1 / 2}\right\}
\end{aligned}
$$

quand

$$
y_{\mathrm{s}}=y_{0} \simeq\left(\frac{\left\langle J J^{*}\right\rangle+\left\langle I_{n 1} I_{n 1}^{*}\right\rangle+y_{1}^{2}\left\langle E E^{*}\right\rangle+2 y_{1}\left\langle E J^{*}\right\rangle}{\left\langle E E^{*}\right\rangle}\right)^{1 / 2} .
$$

L'examen de (11) et (12) montre que $F_{\min }$ et l'admittance optimum $y_{0}$ donnant le facteur de bruit minimum sont des fonctions croissantes de $I_{\mathrm{L}}$. Pour les courants émetteur inférieurs à quelques $\mathrm{mA}$ nous avons déjà mentionné que les deux derniers termes de (11) et (12) sont négligeables.

Dans ce cas, les expressions (11) et (12) se réduisent à :

$$
\begin{gathered}
F_{\min } \simeq 1+\frac{1}{2 k T \Delta f}\left\{\left\langle E J^{*}\right\rangle+y_{1}\left\langle E E^{*}\right\rangle+\left[\left\langle E E^{*}\right\rangle\left(\left\langle J J^{*}\right\rangle+\left\langle I_{n 1} I_{n 1}^{*}\right\rangle\right)\right]^{1 / 2}\right\} \\
y_{0} \simeq\left(\frac{\left\langle J J^{*}\right\rangle+\left\langle I_{n 1} I_{n 1}^{*}\right\rangle}{\left\langle E E^{*}\right\rangle}\right)^{1 / 2}
\end{gathered}
$$


Pour des doses d'irradiation faibles telles que $\Delta I_{\mathrm{L}}<I_{\mathrm{L}}$ et $\Delta I_{\mathrm{L}}<I_{\mathrm{BA}}, F_{\min }$ et $y_{0}$ varient peu. Pour des doses telles que $\Delta L_{\mathrm{L}}>I_{\mathrm{BA}}$ et $\Delta I_{\mathrm{L}}>I_{\mathrm{L}}$ le troisième terme de (13) est proportionnel à $I_{\mathrm{L}}$ tandis que le cinquième est proportionnel à $I_{\mathrm{L}}^{1 / 2}$. Néanmoins aux basses et moyennes fréquences $F_{\min }$ est souvent voisin de l'unité et en conséquence la sensibilité de $F_{\min }$ aux accroissements de $I_{\mathrm{L}}$ est faible. Par contre, $y_{0} \propto I_{\mathrm{L}}^{1 / 2}$, entraîne une plus grande sensibilité de $y_{0}$ aux variations de $I_{\mathrm{L}}$.

Pour des courants émetteurs supérieurs à quelques $\mathrm{mA}$, le facteur de bruit minimum peut être notablement supérieur à l'unité. Sa sensibilité aux variations de $I_{\mathrm{L}}$ sera plus grande que dans le cas précédent puisque la restriction $F_{\min } \simeq 1$ ne s'applique plus. De même, à cause du terme $y_{1}^{2}\left\langle E E^{*}\right\rangle$ qui peut ne pas être négligeable, les variations de $y_{0}$ peuvent croître plus vite que $I_{\mathrm{L}}^{1 / 2}$.

$\mathrm{Ce}$ qui précède s'applique à tous les transistors quelles que soient leurs caractéristiques internes. Toutefois en pratique, l'examen de (1) à (14) indique que pour une dose absorbée donnée de rayonnement, l'amplitude des augmentations du facteur de bruit peut être très différente d'un transistor à un autre. Ce qui distingue deux transistors ce sont les valeurs relatives de $I_{\mathrm{BA}}$ et $I_{\mathrm{L}}$ et plus précisément le rapport $I_{\mathrm{L}} / I_{\mathrm{BA}}$. D'une manière générale plus ce rapport sera élevé et plus les évolutions de $F$ et $y_{0}$ seront de grande amplitude. Autrement dit comme $I_{\mathrm{L}}$ est étroitement lié aux propriétés superficielles, il découle de ce qui précède que les transistors dont la surface est de mauvaise qualité ( $I_{L}$ grand) doivent être les plus sensibles au rayonnement ionisant. Enfin, notons que dans le cas où $I_{\mathrm{L}}$ tend vers une valeur limite consécutive à l'application de doses de rayonnement élevées, les accroissements du facteur de bruit résultant de ceux de $I_{\mathrm{L}}$ doivent être bornés.

A priori, la question qu'il convient de se poser maintenant est : existe-t-il des mécanismes, autres que ceux qui viennent d'être analysés, qui peuvent influencer le comportement en bruit du transistor? L'étude expérimentale qui suit a pour but de répondre à cette question.

3. Etude expérimentale. - 3.1 LES CONDITIONS DE L'ÉTUDE. - Les transistors utilisés pour cette étude sont des transistors bipolaires commandables par effet de champ, parfois désignés par le terme tétrode de Sah. Ils se distinguent des transistors bipolaires usuels par la présence d'une métallisation (électrode) supplémentaire placée sur la couche d'oxyde de silicium recouvrant la jonction émetteur base. L'application d'une tension, dite de grille, et notée dans ce qui suit $V_{G}$, sur cette électrode supplémentaire permet, par effet de champ, de modifier la répartition du potentiel électrostatique à la surface du transistor et donc de simuler la présence de charges électriques fixes dans la couche superficielle d'oxyde. Ces transistors ont été fabriqués au LAAS par diffusion de bore et de phosphore sur un substrat épitaxié $\mathrm{N} / \mathrm{N}^{+}$. Typiquement la résistivité de la base est de $2,6 \times 10^{-2} \mathrm{ohm} . \mathrm{cm}$ avec une concentration en surface de l'ordre de quelques $10^{18}$ atomes d'impuretés de dopage par $\mathrm{cm}^{3}$. Les métallisations sont en aluminium, l'épaisseur de l'oxyde superficiel est de $0,32 \mu \mathrm{m}$. La vitesse de recombinaison en surface est voisine de $20 \mathrm{~cm} / \mathrm{s}$ et la quantité de charges positives dans l'oxyde avant irradiation est de l'ordre de $10^{12}$ atomes $/ \mathrm{cm}^{2}$. Le gain en courant contini: $h_{\mathrm{FE}} \simeq 120$ pour $I_{\mathrm{C}}=1 \mathrm{~mA}$.

La source de rayonnement utilisée est un générateur de rayons $\mathrm{X}$, fabriqué par C.G.R., du type Aequivolt 300. Ce générateur a été utilisé avec une tension d'alimentation de $150 \mathrm{kV}$ et un courant de $10 \mathrm{~mA}$. Dans ces conditions, il délivre une dose de $10^{5} \mathrm{~Gy} \mathrm{~min}^{-1}$. Pendant l'irradiation, les électrodes du transistor sont court-circuitées. Les mesures ont été effectuées immédiatement après irradiation avec des doses comprises entre $10^{5}$ et $10^{8} \mathrm{~Gy}$.

Le facteur de bruit a été mesuré par la méthode des trois mesures [13]. Dans le dispositif mis en œuvre, le transistor a été monté en émetteur commun. Le bruit apparaissant au collecteur a d'abord été amplifié puis filtré et redressé par un voltmètre sélectif modèle H.P. 312 B. Le signal délivré par le voltmètre a été ensuite appliqué à l'entrée d'un intégrateur de grande constante de temps. La valeur du signal résultant a été déterminée par une méthode d'opposition. Pour éviter de changer le calibre du voltmètre sélectif on a utilisé des atténuateurs étalonnés de grande précision. Le gain de la chaîne de mesure a été obtenu avec un générateur de bruit blanc. Le facteur de bruit a été mesuré pour 12 valeurs de la résistance de source comprise entre $10 \mathrm{ohms}$ et $3 \times 10^{5}$ ohms. A partir de ces résultats, la courbe du facteur de bruit en fonction de la résistance de source a été calculée numériquement par un programme de régression qui minimise la valeur quadratique des écarts. L'erreur totale effectuée sur l'estimation des valeurs du facteur de bruit n'excède pas $8 \%$.

D'une manière générale les autres conditions de mesure ont été les suivantes : courant collecteur $I_{\mathrm{C}}=100 \mu \mathrm{A}$, tension collecteur émetteur $V_{\mathrm{CE}}=3 \mathrm{~V}$, température $20^{\circ} \mathrm{C}$, fréquence $f=100 \mathrm{kHz}$. A $100 \mathrm{kHz}$ le spectre de bruit n'a pas montré de dépendance fréquentielle même après avoir soumis les transistors à des doses de $10^{8} \mathrm{~Gy}$.

3.2 Résultats. - Les figures 2 et 3 représentent les variations du facteur de bruit en fonction de la résistance de source $R_{\mathrm{s}}$ pour deux tensions de grille correspondant respectivement à l'existence d'une zone d'accumulation à la surface de base $\left(V_{\mathrm{G}}=-40 \mathrm{~V}\right)$ et à la présence d'une zone d'appauvrissement

$$
\left(V_{\mathrm{G}}=+10 \mathrm{~V}\right) \text {. }
$$

Les différentes courbes montrent le facteur de bruit 


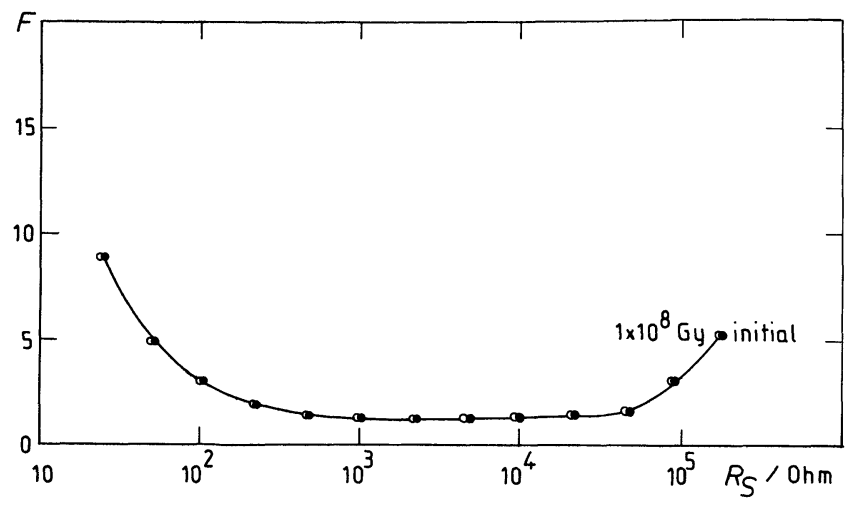

Fig. 2. - Facteur de bruit en fonction de la résistance de source à $V_{\mathrm{G}}=-40 \mathrm{~V}, f=100 \mathrm{kHz}, I_{\mathrm{C}}=100 \mu \mathrm{A}$ et $V_{\mathrm{CE}}=3 \mathrm{~V}$. Les symboles $\bullet$ représentent les valeurs avant irradiation. Les symboles $O$ donnent le facteur de bruit après $10^{8} \mathrm{~Gy}$.

[Noise figure $v s$. source resistance at $V_{\mathrm{G}}=-40 \mathrm{~V}, f=100 \mathrm{kHz}$, $I_{\mathrm{C}}=100 \mu \mathrm{A}, V_{\mathrm{CE}}=3 \mathrm{~V}$. Symbols $\bullet$ are the pre-irradiation values. Symbols $O$ indicate noise figure after $10^{8} \mathrm{~Gy}$.]

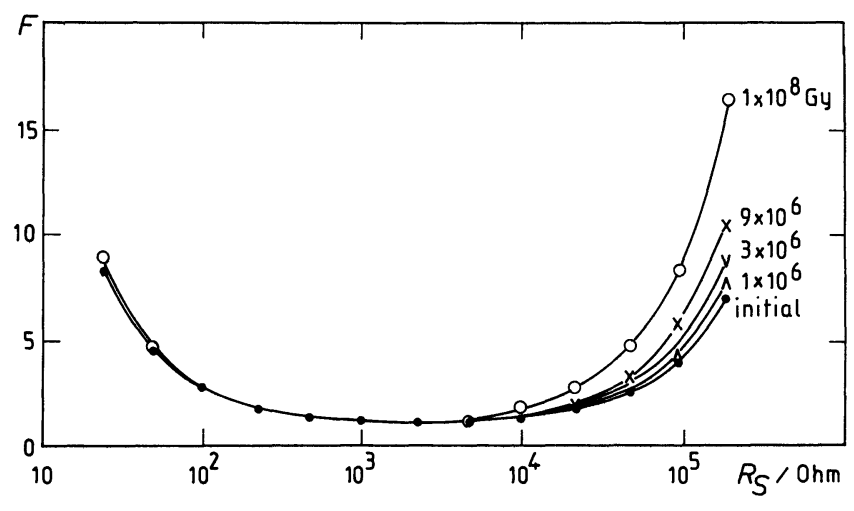

Fig. 3. - Facteur de bruit en fonction de la résistance de source à $V_{\mathrm{G}}=+10 \mathrm{~V}, f=100 \mathrm{kHz}, I_{\mathrm{C}}=100 \mu \mathrm{A}$ et $V_{\mathrm{CE}}=3 \mathrm{~V}$. Les symboles donnent les valeurs avant irradiation, $\wedge$ après $10^{6} \mathrm{~Gy}$, $\checkmark$ après $3 \times 10^{6} \mathrm{~Gy}, \times$ après $9 \times 10^{6} \mathrm{~Gy}$, et $O$ après $10^{8} \mathrm{~Gy}$.

[Noise figure vs. source resistance at $V_{\mathrm{G}}=+10 \mathrm{~V}, f=100 \mathrm{kHz}$, $I_{\mathrm{C}}=100 \mu \mathrm{A}, \quad V_{\mathrm{CE}}=3 \mathrm{~V}$. Symbols $\bullet$ are the pre-irradiation values, $\wedge$ after $10^{6} \mathrm{~Gy}, \vee$ after $3 \times 10^{6} \mathrm{~Gy}, \times$ after $9 \times 10^{6} \mathrm{~Gy}$ and $O$ after $10^{8} \mathrm{~Gy}$.]

avant irradiation et ses évolutions pour des doses absorbées égales respectivement à :

$$
\begin{aligned}
& 1 \times 10^{6} \mathrm{~Gy}, 3 \times 10^{6} \mathrm{~Gy}, \\
& 9 \times 10^{6} \mathrm{~Gy} \text { et } 1 \times 10^{8} \mathrm{~Gy} .
\end{aligned}
$$

L'examen de ces figures permet d'effectuer les observations suivantes :

a) pour des résistances de source $R_{\mathrm{s}}<10^{3} \mathrm{ohms}$, le facteur de bruit est constant quelle que soit la dose absorbée et quel que soit l'état électrique de la surface de la base,

b) pour des résistances de source supérieures à $10^{4} \mathrm{ohms}$, le facteur de bruit augmente avec la dose.
Les accroissements correspondant à l'existence d'une zone d'appauvrissement à la surface de la base sont nettement plus importants que ceux associés à la présence d'une zone d'accumulation (ces derniers sont très faibles et n'ont pas été représentés),

c) la résistance donnant le facteur de bruit minimum $R_{\mathrm{s} 0}$ diminue quand la dose de rayonnement absorbée augmente. Sur les figures 4 et 5 sont portées les variations du facteur de bruit et de l'admittance $y_{0}=1 / R_{\mathrm{s} 0}$ en fonction du courant de base pour $V_{\mathrm{G}}=0 \mathrm{~V}$. Ce cas est analogue à celui d'un transistor bipolaire usuel puisque le potentiel électrostatique à l'interface $\mathrm{Si}_{-} \mathrm{SiO}_{2}$ ne dépend que de la charge électrique située dans l'oxyde. Les croix représentent les valeurs initiales, les triangles et les cercles sont les valeurs après irradiation. Le facteur de bruit minimum (triangles) reste voisin de l'unité. Ses évolutions sont du même ordre de grandeur que les erreurs de mesure. Par contre le facteur de bruit pour une résistance de source de $180 \mathrm{kohms}$ (cercles) et l'admittance $y_{0}$ sont des fonctions croissantes du courant de base. Pour préciser ce comportement et le comparer à celui obtenu à la section 2.3 , les accroissements du facteur de bruit et du carré de l'admittance de source donnant le facteur de bruit minimum ont été portés en fonction des accroissements de la composante superficielle du courant de base. Les résultats sont montrés sur les figures 6 et 7 . Aux erreurs expérimentales près, $F \propto \Delta I_{\mathrm{L}}$ et $\Delta\left(y_{0}^{2}\right) \propto \Delta I_{\mathrm{L}}$. Des comportements analogues ont été mis en évidence sur tous les transistors étudiés.

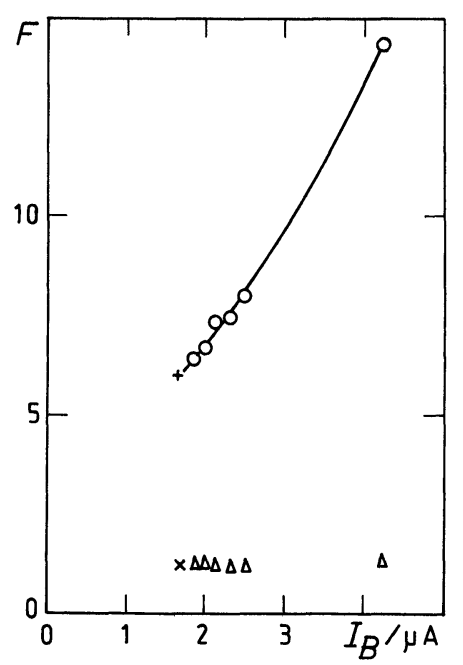

Fig. 4. - Facteur de bruit en fonction de la composante superficielle du courant de base à $V_{\mathrm{G}}=0 \mathrm{~V}, f=100 \mathrm{kHz}, I_{\mathrm{C}}=100 \mu \mathrm{A}$ et $V_{\mathrm{CE}}=3 \mathrm{~V}$. Les symboles + et $\times$ représentent les valeurs initiales. Les symboles $\Delta$ sont le facteur de bruit minimum après plusieurs doses d'irradiation. Les symboles $O$ indiquent le facteur de bruit obtenu avec une résistance de source de 180 kohms.

[Noise figure vs. base surface current at $V_{\mathrm{G}}=0 \mathrm{~V}, f=100 \mathrm{kHz}$, $I_{\mathrm{C}}=100 \mu \mathrm{A}, V_{\mathrm{CE}}=3 \mathrm{~V}$. Symbols + and $\times$ represent initial values. Symbols $\Delta$ are the minimum noise figure after various radiation doses. Symbols $O$ indicate the noise figure obtained with a $180 \mathrm{kohms}$ source resistance.] 


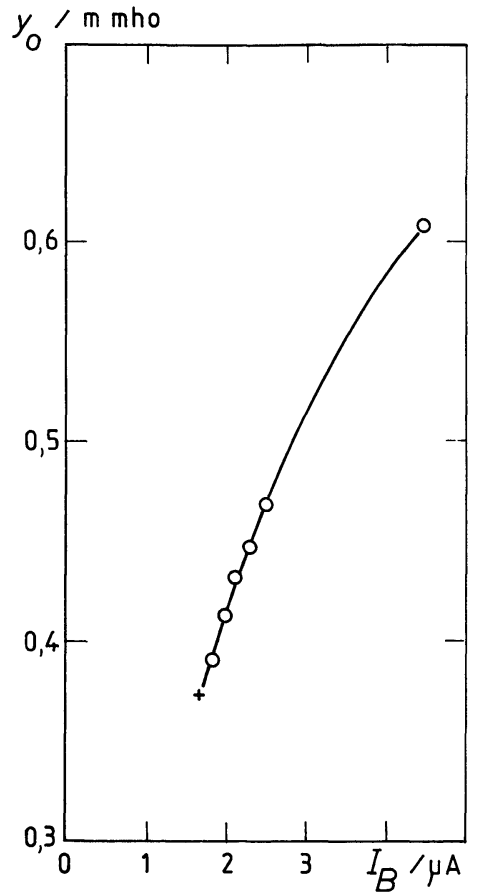

Fig. 5. - Admittance associée au facteur de bruit minimum en fonction du courant de base à $V_{\mathrm{G}}=0 \mathrm{~V}, f=100 \mathrm{kHz}, I_{\mathrm{C}}=100 \mu \mathrm{A}$ et $V_{\mathrm{CE}}=3 \mathrm{~V}$. Le symbole + indique la valeur avant irradiation, les symboles $O$ celles après différentes doses d'irradiation.

[Minimum noise figure admittance vs. base current at $\iota_{G}=0 \mathrm{~V}$, $f=100 \mathrm{kHz}, I_{\mathrm{C}}=100 \mu \mathrm{A}, V_{\mathrm{CE}}=3 \mathrm{~V}$. Symbol + represents the pre-irradiation value, symbol $\bigcirc$ after various radiation doses.]

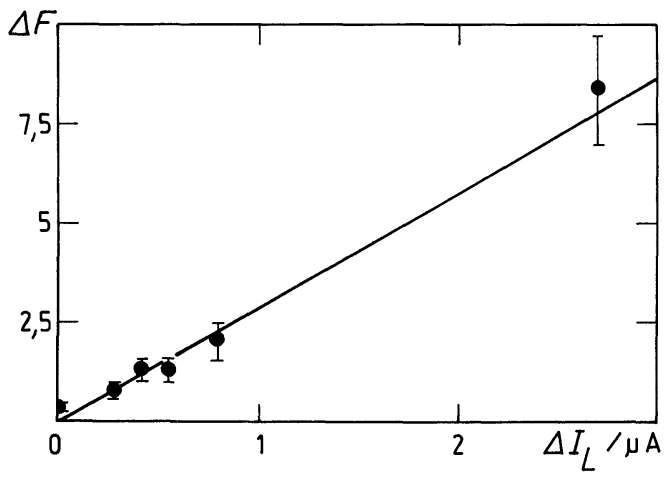

Fig. 6. - Accroissements du facteur de bruit en fonction des accroissements de la composante superficielle du courant de base à $V_{\mathrm{G}}=0 \mathrm{~V}, f=100 \mathrm{kHz}, I_{\mathrm{C}}=100 \mu \mathrm{A}, V_{\mathrm{CE}}=3 \mathrm{~V}$. La précision est indiquée par les symboles $\mathrm{I}$.

[Noise figure increases $v s$. surface base current increases at $V_{\mathrm{G}}=0 \mathrm{~V}$, $f=100 \mathrm{kHz}, I_{\mathrm{C}}=100 \mu \mathrm{A}, V_{\mathrm{CE}}=3 \mathrm{~V}$. Symbols I indicate accuracy.]

On voit que l'ensemble de ces observations sont en bon accord avec le modèle développé dans la section 2.3. Ceci implique que :

a) les évolutions du facteur de bruit et de ses paramètres $F_{\min }$ et $y_{0}$, sont complètement déterminées par celles de la composante superficielle du courant de base ;

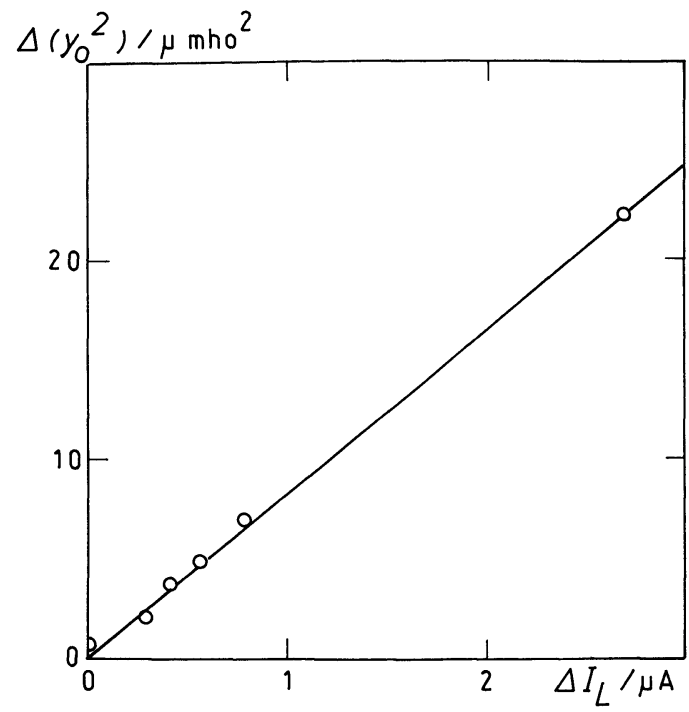

Fig. 7. - Dérives dues à l'irradiation du carré de l'admittance de source optimum en fonction des accroissements de la composante superficielle du courant de base, à $V_{\mathrm{G}}=0 \mathrm{~V}, f=100 \mathrm{kHz}$, $I_{\mathrm{C}}=100 \mu \mathrm{A}$ et $V_{\mathrm{CE}}=3 \mathrm{~V}$.

[Radiation shifts of the square of the optimum admittance $v s$. surface base current increases at $V_{\mathrm{G}}=0 \mathrm{~V}, f=100 \mathrm{kHz}$, $I_{\mathrm{C}}=100 \mu \mathrm{A}, V_{\mathrm{CE}}=3 \mathrm{~V}$.]

b) le bruit en excès observé aux moyennes fréquences après irradiation provient des centres de génération recombinaison créés par le rayonnement ionisant dans les zones superficielles de la jonction émetteur base.

Ainsi, l'étude expérimentale du bruit de fond aux moyennes fréquences confirme que le rayonnement ionisant n'a aucun effet sur les propriétés volumiques du transistor et que par contre il dégrade significativement les caractéristiques superficielles. L'absence de modèle précis reliant ces dernières au bruit de fond ne permet pas actuellement de déduire d'une étude détaillée du bruit les évolutions des paramètres de surface. Toutefois l'analyse de la signification et des implications des différences de comportement quand il existe une zone d'accumulation ou d'appauvrissement à la surface de la base donne des informations d'un grand intérêt pratique. Rappelons que ce qui différencie ces deux états c'est l'étendue de la zone de charge d'espace de la jonction émetteur base à la surface. L'augmentation notable du bruit de fond après irradiation quand la jonction s'étend (régime d'appauvrissement), signifie que les centres de génération recombinaison sont créés sur toute la surface, non seulement dans la zone occupée par la jonction quand le transistor est soumis au rayonnement mais aussi en dehors. Or, il a déjà été mentionné que l'étendue de la charge d'espace superficielle était contrôlée dans les transistors sans grille par la quantité de charges électriques existant ou créée dans la couche d'oxyde. Si les évolutions de la charge électrique tendent à provoquer une extension de la 
jonction, la sensibilité du bruit au rayonnement sera élevée. Par contre si la charge créé tend à accumuler la surface de la base, la sensibilité sera faible. Cette analyse sommaire des problèmes de sensibilité ne prend pas en compte l'existence possible de répartitions hétérogènes des charges et des centres de génération recombinaison. $\mathrm{Si}$ des distributions de ce type se produisent, les tendances générales précédentes peuvent être partiellement masquées. Des expériences complémentaires sont nécessaires pour élucider ces effets.

4. Conclusion. - Les évolutions du facteur de bruit des transistors bipolaires aux moyennes fréquences résultant des accroissements des composantes superficielles du courant de base induits par un rayonnement ionisant ont été analysées et comparées à celles observées sur des transistors exposés au rayonnement $\mathrm{X}$ délivré d'une manière continue par un générateur fonctionnant à $150 \mathrm{kV}, 10 \mathrm{~mA}$. En dessous de $10^{5} \mathrm{~Gy}$, il n'a pas été noté d'effets significatifs des rayons $X$ sur le facteur de bruit. Pour des doses supérieures, il ressort de l'analyse et de l'expérience que :

a) le facteur de bruit est constant pour une résistance de source faible inférieure à la résistance donnant le facteur de bruit minimum,

$b$ ) pour des résistances de source élevées, le facteur de bruit augmente, ses accroissements sont proportionnels à ceux de la composante superficielle du courant de base $I_{\mathrm{L}}$,

c) les accroissements du carré de l'admittance donnant le facteur de bruit minimum sont proportionnels aux accroissements de $I_{\mathrm{L}}$.

Autrement dit, les évolutions du facteur de bruit sont entièrement déterminées par celles de $I_{\mathrm{L}}$.

De plus, il a été également montré que la sensibilité du facteur de bruit au rayonnement dépendait de l'état de la surface. Quand la surface de la base est en régime d'accumulation, l'amplitude des accroissements du facteur de bruit est très faible même après $10^{8} \mathrm{~Gy}$. Par contre, quand la surface est appauvrie de porteurs majoritaires, les dégradations du facteur de bruit peuvent être très importantes.

$\mathrm{Au}$ plan des applications, les résultats présentés dans cet article montrent que dans les circuits électriques, il est préférable d'utiliser des résistances de source inférieures, ou au plus égales, à celles donnant le facteur de bruit minimum. Dans les cas où l'on n'a pas le choix de l'impédance de source et où elle a une valeur élevée, il est souhaitable d'utiliser des composants durcis. Au plan technologique, il découle de ce qui précède que les traitements et règles de conception des transistors qui sont susceptibles de minimiser la sensibilité du bruit aux moyennes fréquences sont ceux qui minimisent la sensibilité du gain en courant au rayonnement.

Remerciements. - Le banc de mesure du facteur de bruit a été conçu et réalisé par J. Caminade. Les transistors commandables par effet de champ ont été fabriqués avec l'aide de G. Pierrel, J. Chevalier, F. Rossel et P. Fadel.

Les auteurs les en remercient très vivement.

\section{Bibliographie}

[1] Snow, E. H., Grove, A. S. and Fitzgerald, D. J., Proc. I.E.E.E. 55 (1967) 1168

[2] KoII, E., I.E.E.E. Trans. ED 13 (1966) 12.

[3] Hughes, H. L., I.E.E.E. Trans. NS 16 (1969) 195.

[4] Revesz, A. G., ZaIninger, K. H., RCA Rev. 3 (1968) 22.

[5] Buxo, J., Caractérisation expérimentale et analyse de l'effet de rayonnement ionisant sur les propriétés électriques des transistors M.O.S., Thèse de Doctorat ès Sciences Physiques (Université P. Sabatier de Toulouse) 1972.

[6] VAN VliET, K. M., I.E.E.E. Trans. ED 23 (1976) 1236.

[7] Martin, J. C., Blasquez, G. et Caminade, J., Electron. Lett. 7 (1971) 342.

[8] Rey, G. et LetURCQ, Ph., Théorie approfondie du transistor bipolaire (Masson, Paris) 1972, p. 112.
[9] Martin, J. C., Blasquez, G. et Caminade, J., Le bruit de fond des composants actifs semi-conducteurs. Colloque International du C.N.R.S. $\mathrm{n}^{\mathrm{o}} 204$ (C.N.R.S., Paris) 1971, p. 49.

[10] Blasquez, G., Caminade, J. et Le Gac, G., Physica 92B + C (1977) 313.

[11] GARRIC, J. C., Caractérisation des composantes du courant de base dans les transistors bipolaires plans. Thèse de Doctorat de $3^{\mathrm{e}}$ cycle (Université Paul-Sabatier de Toulouse) 1974.

[12] Blasquez, G., Caminade, J. et Le Gac, G., Physica 94B + C (1978) 359.

[13] VAN DER ZIEL, A., Noise (Prentice-Hall Inc., Englewood (liffs, N.J.)), 1970, p. 30. 